\title{
Invalid results from the method of constant stimuli
}

MOSHE LEVISON AND FRANK RESTLE INDIANA UNIVERSITY

The Method of Constant Stimuli was shown to produce constant errors in the direction expected by $E$. In an experiment, 89 Ss compared variable lines with a standard, each $S$ producing a psychometric function and Point of Subjective Equality (PSE). Four groups differed in the particular range of variable stimuli used; whether the stimuli averaged longer or shorter than the standard, and whether they had a wide or narrow range. All sets of variable stimuli encompassed the standard. PSE was consistently between the standard and the mean of the variables, as predicted by Adaptation Level Theory. In many experiments, the set of variables is chosen on the basis of theory or pilot studies; the present study shows that PSE will be spuriously drawn toward the center of the variables, hence toward $E^{\prime}$ s pre-experimental expectations.

Woodworth and Schlosberg (1954) stated several advantages of the Method of Constant Stimuli (MCS). (1) The Method of Average Error is impractical in some fields, because the stimulus is not readily adjusted by $O$ or E. MCS uses only fixed stimuli. (2) MCS avoids errors of habituation and anticipation, which occur in the Method of Limits. (3) MCS uses all responses in the psychometric function, whereas the Method of Limits only considers transition points. (4) Although MCS uses many trials, each trial takes very little time. MCS yields a psychometric function from which both the Point of Subjective Equality (PSE), the median of the function, and the differential limen (DL), the probable error of the function, can be estimated. The same authors advise that the experiment should be carefully planned; a pilot study is used to select a series of stimuli that adequately covers $O$ 's transition zone.

In practical application of the Method of Constant Stimuli, E is faced with the need to select a distribution of variable stimuli. Applications of Adaptation Level Theory (Helson, 1964) to this experimental situation suggest that the choice of the variable stimuli might have a detectable effect on the outcome of the experiment. The present experiment was designed to test whether measurements of the length of lines (a simple and relatively easy discrimination) could be affected by moderate variations in the distribution of variable stimuli.

METHOD
Subjects
Ninety-five male and female students enrolled in introductory
psychology courses at Indiana University participated in the
experiment to fulfill a course requirement. Subjects were run in
groups of four, and each group was randomly assigned to one of
the four conditions.

\section{Apparatus and materials}

Stimuli were slides of white lines at $45 \mathrm{deg}$ projected on a darkened screen in a moderately dark room. The slides were projected, from a Spindler-Saupe random access projector outside the experimental chamber through a window located above and behind the Ss, onto a $4 \times 4 \mathrm{ft}$ beaded glass screen. The standard slide presented to all groups was $8.25 \mathrm{in}$. long. The variable comparison lines used for the various conditions are shown in Table 1. The spacings are somewhat irregular, but the median of the High range sets is $9.00 \mathrm{in}$. in both sets, and the medians of the Low sets are 7.00 and 7.50 . The difference between the longest and shortest variable line is 5.25 and 4.50 for the two wide sets, High and Low; the differences for the narrow sets are both 2.75 . The irregularities did not greatly disturb the intended experimental variables. The standard stimulus at 8.25 in. was within the range of the variables in all conditions; between slides 2 and 3 of the High sets, and between slides 5 and 6 of the Low sets.
Table 1

Lengths of Comparison Lines in Four Groups (as measured at screen in in.)

\begin{tabular}{|c|c|c|c|c|c|c|c|c|}
\hline Group & & & Leng & hs Use & & & & Mean \\
\hline High Wide & 6.75 & 7.50 & 8.50 & 9.00 & 10.25 & 11.13 & 12.00 & 9.30 \\
\hline Low Wide & 4.50 & 5.50 & 6.25 & 7.00 & 7.75 & 8.50 & 9.00 & 6.93 \\
\hline High Narrow & 7.50 & 7.75 & 8.50 & 9.00 & 9.00 & 9.94 & 10.25 & 8.85 \\
\hline Low Narrow & 6.25 & 6.75 & 7.00 & 7.50 & 7.75 & 8.50 & 9.00 & 7.54 \\
\hline
\end{tabular}

\section{Procedure}

On each trial the $\mathrm{S}$ was first presented with the standard slide for $2 \mathrm{sec}$; a variable stimulus was presented $2.7 \mathrm{sec}$. later and remained in view for $2 \mathrm{sec}$. While the variable was present and for $2 \mathrm{sec}$ thereafter, Ss were allowed to respond by pressing the appropriate button on the panel in front of them to indicate that the variable was judged larger or smaller than the standard. The ITI was $5 \mathrm{sec}$. The session consisted of 19 trials of seven comparisons each, with each variable presented once per trial in random order. There was a 30 -sec break half way through the session. The results of the last 15 trials were included in the data. All programming of stimuli, presentation of slides and data collection were done by an IBM 1800 on-line computer.

\section{RESULTS}

Data from six Ss were discarded because their psychometric functions were not monotonic, and it was therefore difficult to estimate their values of PSE. PSE was calculated for each of 89 remaining Ss, and mean PSEs were calculated for each condition. Mean PSEs for the two High conditions (High-Wide and High-Narrow) were above the standard, showing a positive time-order effect (TOE). Mean PSEs for the two Low conditions (Low-Wide and Low-Narrow) were below the standard, a negative TOE (see Table 2). The difference between mean PSEs of the combined High vs combined Low conditions was significant at the .002 level ( $t$ test); the differences between the Wide and Narrow conditions were not significant.

For every condition, the mean PSE was between the standard and the arithmetic mean of the variable stimuli. The means of the variable stimuli in the High-Narrow vs Low-Narrow conditions differed by 1.31 in., and the mean observed PSEs differed by $0.26 \mathrm{in}$. or $19.8 \%$. The means of the variable stimuli in the High-Wide vs Low-Wide conditions differed by 2.38 in., and the mean PSEs differed by 0.42 in., or $17.6 \%$. Shifts of PSE as great as $17-20 \%$ are as large as many visual illusions and other perceptual effects of small magnitude but of relative theoretical importance.

\section{DISCUSSION}

The range of variable stimuli has a surprisingly large and consistent effect upon the location of PSE. The effect is surprising because one expects Ss to judge straight lines accurately when no illusion-inducing fields are present. Although the magnitude of the effect was not very large, it was great enough to have a serious

Table 2

Mean PSE as a Function of Variable Stimuli

\begin{tabular}{lcc} 
Group & N & $\begin{array}{c}\text { Observed } \\
\text { Mean PSE }\end{array}$ \\
\hline High Wide & 24 & 8.35 \\
Low Wide & 20 & 7.93 \\
High Narrow & 22 & 8.33 \\
Low Narrow & 23 & 8.06 \\
\hline
\end{tabular}


effect on the interpretation of experimental data, especially if mathematical functions are to be fit to data points.

According to Helson (1964), S can be thought to rate the comparison stimulus relative to a comparison adaptation level (CAL), and PSE is that stimulus rated at CAL. Let X stand for the length of the comparison stimulus, $S$ stand for the standard, $\bar{X}$ stand for the mean of the comparison stimuli, and $K$ for all constant residual factors. Then in general,

$$
\mathrm{CAL}=\left[\mathrm{X}^{\mathrm{W}_{1}} \mathrm{~S}^{\mathrm{W}_{2}} \mathrm{X}^{\mathrm{W}_{3}} \mathrm{~K}^{\mathrm{W}_{4}}\right] 1 / \mathrm{w}_{1}+\mathrm{w}_{2}+\mathrm{w}_{3}+\mathrm{w}_{4}
$$

where $w_{1}, w_{2}, w_{3}$, and $w_{4}$ are the relative weights of the comparison stimulus (self-adaptation), the standard, the series effect, and constant factors, respectively. If $\mathrm{w}_{3}$ is not negligible, the effect observed in the present experiment should be predicted.

Rewrite Equation 1 as a function only of $\overline{\mathrm{X}}$, averaging over other factors, here represented by $\mathrm{K}$.

$$
\mathrm{CAL}_{\text {high }}=\mathrm{K}\left(\overline{\mathrm{X}}_{\text {high }}\right)^{\mathrm{W}_{3}}
$$

and

$$
\mathrm{CAL}_{\text {low }}=\mathrm{K}\left(\overline{\mathrm{X}}_{\text {low }}\right)^{\mathrm{w}_{3}}
$$

Dividing,

$$
\mathrm{CAL}_{\text {high }} / \mathrm{CAL}_{\text {low }}=\left(\overline{\mathrm{X}}_{\text {high }} / \overline{\mathrm{X}}_{\text {low }}\right)^{\mathrm{w}_{\mathbf{3}}}
$$

Mean CALhigh is 8.34, and mean CALlow is 8.00 . The means of the, variable stimuli are, averaging over wide and narrow, $\bar{X}_{\text {high }}=$ $9.00, \bar{X}_{\text {low }}=7.25$. Inserting these values in Equation 3,

$$
1.240=(1.043)^{\mathrm{W}_{3}}
$$

whence $w_{3}=.200$.
This can be interpreted to signify that the mean of the distribution of variables contributes .20 , or one-fifth, of the total of factors controlling the response.

These results show how the Method of Constant Stimuli may lead an unwary investigator into a wrong conclusion. Theory, previous results, or a brief pilot stydy may yield expectations of where PSE will be found. An experimenter, having decided to make accurate and valid measurements despite the extra effort, decides to employ the method of constant stimuli. He prepares comparison stimuli centering around the expected values of PSE. Our results show that he will then collect data that are spuriously drawn toward the pre-experimental expectations he may have formed. If those expectations were wrong or oversimplified, his experiment will nevertheless tend to give them exact quantitative verification; and since his verifying data were collected in a most laborious and respectable fashion, his results will be convincing both to the experimenter and to his sophisticated readers. This pattern of methodological error seems to be about the most dangerous possible.

\section{REFERENCES}

HELSON, H. Adaptation Level Theory; An experimental and systematic approach to behavior. New York: Harper and Row, 1964.

WOODWORTH, R. S., \& SCHLOSBERG, H. Experimental psychology. (Rev. ed.), New York: Henry Holt, 1954.

NOTE

1. Supported by NSF Grant GB 5714 and NIH Grant MH 12541.

(Accepted for publication March 28, 1968.) 